\title{
APROXIMACIÓN TEÓRICA AL FENÓMENO DEL SUBDESARROLLO 1
}

\section{Xabier Arrizabalo Montoro2}

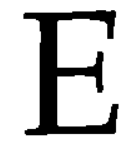

ste artículo es el segundo de una serie de tres en los que abordamos cómo enfocar el estudio del subdesarrollo. En él, no presentamos un análisis exhaustivo sobre todos los aspectos del subdesarrollo, sino una mera aproximación teórica al fenómeno.

Se estructura sobre la base de exponer, en primer lugar, la génesis histórica -origen y formación- del subdesarrollo, es decir, su conformación. Seguidamente, se trata de caracterizar su estructura a partir del estudio de sus rasgos principales. Junto a ello, lo conceptualizaremos con cierta "precisión" - ya que sólo habremos visto hasta entonces sus expresiones y su sustrato subyacenteConcluiremos esta parte abordando el fenómeno de forma interpretativa a través de la consideración de su dinámica de reproducción ${ }^{3}$. Finalizamos el artículo esbozando someramente una discusión clásica como es la de los indicadores de desarrollo.

1. El origen del subdesarrollo: la expansión del modo de producción capitalista

$\mathrm{F}^{\mathrm{l}}$ l origen de lo que conocemos como fenómeno del subdesarrollo Laparece ligado a la conformación del modo de producción capitalista y a la expansión mundial del mismo. 
El modo de producción capitalista se conforma históricamente a partir de la acumulación originaria de capital. Ésta tiene su fundamento en el excedente generado en la producción industrial manufacturera. Excedente consolidado con base en la revolución agrícola y en el paso de la producción manufacturera artesanal a la industrial (máquinas, fábricas, etcétera), así como en la apropiación de excedente de otras formaciones sociales no capitalistas. Todos estos cambios, que están en la explicación de la acumulación originaria del capital, tienen lugar entre los siglos XVI y XVIII —al respecto, véase el excelente capítulo XXIV de Marx (1984) -

Su expansión obedece a la lógica del capital de maximización de la ganancia, para lo cual resulta funcional ta difusión exterior. Esta expansión tuvo lugar históricamente a lo largo de dos grandes etapas que, a su vez, pueden ser divididas en distintas fases.

La primera es la del capitalismo comercial. Transcurre, grosso modo, entre mediados del siglo XVI y finales del XIX. Predomina el capital industrial. Todavía es, en cierta medida, un capitalismo de tipo concurrencial en el gue la competencia desempeña un papel tanto cuantitativa como cualitativamente distinto del que jugará en la etapa siguiente caracterizada por la tendencia al monopolio - lo cual no anulará, en todo caso, la competencia intercapitalista-. Su expansión consiste en el intercambio de mercancías (productos primarios de los países actualmente subdesarrollados por manufacturados de los desarrollados) que conforma una división internacional del trabajo.

En la segunda, el capitalismo es de tipo monopólico. Se extiende desde finales del siglo XIX hasta la actualidad. Es la etapa imperialista del capitalismo, caracterizada por el predominio del capital financiero, (derivado de la fusión del capital bancario con el industrial, en la que aquel tiende a predominar - $\mathrm{a}$ imponerse- en el tiempo sobre éste). Además de persistir la expansión comercial, se consolida la exportación de capital (inversión en cartera) hacia los países subdesarrollados y se profundiza la división internacional del trabajo apuntada anteriormente. Las empresas transnacionales desempeñan un importante rol como sujetos de la internacionalización del capital, que también se da en la esfera productiva (inversión directa). El capitalismo ha perdido definitivamente todo el carácter progresivo que podía haber tenido ante- 
riormente Su estudio será abordado con mayor detenimiento en el tercer artículo de esta serie.

Por tanto, es a partir de la expansión del modo de producción capitalista como se conforma la estructura del subdesarrollo. Como señala Ramos (1980b; 5, subrayado del autor) "no se trata por lo tanto, ni de atraso, ni de incapacidad natural o racial, ni de escasez de recursos, sino de un proceso de subordinación a la expansión comercial y financiera del capitalismo, que por otra parte no es idílico, sino que va ligado a los cañones de las armadas imperiales, además de a la mayor productividad de sus industrias". De manera que la evolución de estos países será inducida desde el exterior, a diferencia de la de los desarrollados en que ésta es autónoma, o al menos, según los casos, autónoma en mayor medida. La inevitable - importante- discusión acerca del papel que juegan los factores internos y los externos, en relación a la consolidación del subdesarrollo como crónico y estructural, se aplaza hasta el momento en que abordemos la reproducción del subdesarrollo que es donde verdaderamente cobra pleno sentido.

Conviene recordar en este momento, siquiera sea brevemente, algunas de las que constituyen las tendencias fundamentales del desarrollo capitalista y, por ende, de su proceso de expansión. Son la centralización y concentración del capital, su evolución cíclica4, fluctuante y contradictoria, su propensión a la internacionalización creciente y el desarrollo desigual de las distintas áreas capitalistas por las relaciones de dominación-subordinación existentes entre ellas.

\section{La estructura del subdesarrollo: caracterización}

T a caracterización de la estructura del subdesarrollo es una taLrea que vamos a comenzar desde una perspectiva eminentemente descriptiva, aplazando su interpretación que se abordará, especialmente, en el terreno del análisis de la dinámica y de la modalidad que toma su reproducción en el tiempo. La confusión de ambos planos por parte de algunos autores y escuelas es lo que lleva a Ramos $(1980 \mathrm{~b} ; 6)$ a señalar, acertadamente, que sus "resultados [los del subdesarrollo] son sólo las manifestaciones empíricas que la teoría convencional toma como la 'explicación" del subdesarrollo". 
Antes de proceder a efectuar dicha caracterización conviene insistir en un punto. Como se dijo, las FSCS resultan ser un conjunto muy heterogéneo. Por ello, aquí nos referimos a un tipo genérico de país al que denominamos subdesarrollado.

Y también previamente, ofreceremos una definición de subdesarrollo que posteriormente, tras la caracterización de su estructura, será retomada para la discusión. En principio, como punto de partida, parece correcta la que propone Ramos (1980b; 16), para quien "el subdesarrollo como concepto teórico es la interrelación de una estructura interna distorsionada por la penetración del capitalismo exterior, con una serie de factores externos interconectados también entre sí y que son el resultado de la posición subordinada y asimétrica de los países subdesarrollados en la división internacional del trabajo, impuesta por los países desarrollados a lo largo de la historia". No obstante, pensamos que debe ser complementada añadiendo que este fenómeno del subdesarrollo, conceptualizado como parte del proceso histórico global de desarrollo del capitalismo, que supone una estructura económica distorsionada y una transferencia de recursos de las FSCS a las FSCD —realizada mediante tasas mayores de explotación en aquellas-, tiene como consecuencia fundamental la de mantener a una parte mayoritaria de su población en unas muy deficientes condiciones de vida, que les impiden la satisfacción de sus necesidades básicas. De modo que la dimensión esencial del subdesarrollo en su expresión capitalista es su carácter clasista5.

Hay que hacer notar que en esta definición no se habla de factores internos y factores externos sino de la interrelación de una "estructura interna distorsionada" con una serie de "factores externos". Esta aclaración no es baladí sino que se constituye en un elemento central para evitar confusiones a la hora de explicar el subdesarrollo. Efectivamente, la definición nos da el punto de partida para señalar que en la interrelación de la estructura interna distorsionada - el conjunto de la estructura económica de estos países- con los factores externos, son todos los aspectos de esa estructura los que son afectados. En términos dinámicos, dichos factores externos interesan a cada una de las dos esferas del proceso económico: producción y circulación. Es decir, no es un solo 
aspecto de esa estructura -o una sola esfera o fase del procesosino su globalidad, la que se ve afectada decisivamente por ese tipo de factores.

De manera que tenemos, por un lado, que el origen del subdesarrollo se encuentra en los efectos para estas sociedades de la expansión del modo de producción capitalista, y, por otro lado, que su explicación radica en una estructura económica deformada - en la que influyen fuertemente elementos externos- que tiende a reproducirse en el tiempo a lo largo del proceso económico.

Así pues, si bien, efectivamente, el subdesarrollo aparece influido por factores externos, no se puede hablar de que dicha influencia tenga lugar globalmente sino a través de cada aspecto y de cada fase del proceso. Es decir, el subdesarrollo no consiste en unas determinadas estructuras productivas y distributivas internas independientes que se ven afectadas por una inserción exterior dada, sino que su esencia es la concreción distorsionada del conjunto del proceso económico que se ve afectado en cada aspecto -en cada fase- por una serie de factores externos. Factores externos que se explican en la lógica de acumulación capitalista que opera a escala mundial y que tienen que ver con el tipo de participación de estas economías en la división internacional del trabajo.

Por esto se explica el hecho de que sean dominadas desde fuera por los centros de la economía capitalista mundial, determinando niveles de dependencia cuantitativa y cualitativamente distintos a los que, eventualmente, pueden sufrir las FSCD6. Dicha dependencia tiene lugar en varios planos que serán abordados al tratar los aspectos concretos a los que afecta cada uno de ellos7 y es la que permite conceptualizar a estas economías como extravertidas.

Por lo tanto, la forma en que vamos a caracterizar el subdesarrollo como un tipo específico de formación social capitalista, será a través del análisis de su particularidad en cada una de las fases del proceso económico. Fases que, lógicamente, están estrechamente interrelacionadas entre sí, de manera que si se analizan por separado, no es porque así aparezcan en la realidad, sino a los efectos, únicamente, de lograr una mayor claridad expositiva. $Y$, en el próximo epígrafe, observándolas conjuntamente en su se- 
cuencia dinámica, abordaremos su reproducción en el tiempo.

Empezaremos tratando la esfera de la producción. En relación a ella se deben citar varios elementos. En primer lugar, la estructura productiva aparece fuertemente desarticulada intersectorialmente. Se da una importante participación del sector primario, debido en gran medida a la conformación primario-exportadora de su inserción exterior - lo cual no permite, sin embargo, asegurar la subsistencia- y un sector terciario excesivamente inflado. El sector industrial presenta escasa importancia relativa ${ }^{8}$ con, como en el conjunto de los sectores, bajas tasas de productividad -los subsectores con mayores tasas suelen estar en manos del capital extranjero-, exigua diversificación y minoritaria orientación al mercado interno. Añadidamente, se da una fuerte tendencia, creciente, al oligopolio.

Vinculado a todos estos elementos, aparecen los fenómenos del desempleo y subempleo masivos -unidos a la baja cualificación de la mano de obra- con lo que implican, en esta esfera, de desaprovechamiento de recursos. Como veremos más adelante, este desaprovechamiento de recursos del lado del trabajo se agudiza y complementa con una tasa de inversión muy pequeña, consecuencia de la existencia de una tasa de ahorro reducida y una menor aún canalización hacia su uso productivo - por cuanto una parte importante de ella es de carácter especulativo-.

La estructura productiva se encuentra fuertemente condicionada por factores externos, manifestándose claramente en este terreno la influencia del fenómeno de la dependencia. El principal ámbito en el que se manifiesta ésta, respecto a la producción, es en el de la dependencia comercial. Consiste, básicamente, en que la producción principal para exportación - de tipo primario, en tanto que las principales importaciones procedentes de los países desarrollados corresponden a productos industriales-, no está integrada en una estructura productiva autocentrada. Ello genera una desarticulación de la estructura productiva interna y un intercambio desigual expresado a través del deterioro de los términos del mismo. De manera que la clase capitalista dominante de las FSCD se apropia de una parte de la plusvalía extraída a la clase trabajadora en las FSCS9. 
También influye la dependencia de carácter financiero que tiene que ver con el hecho diferencial de las FSCS respecto de las FSCD, en cuanto al origen de lo que constituye el núcleo central del financiamiento de la inversión $-\mathrm{y}$, también, en cuanto a dónde se decide el destino que se le da a la inversión-. De modo que, mientras en los desarrollados ésta se obtiene $y$, sobre todo, maneja internamente, en los subdesarrollados se dirige desde el exterior -recurriendo incluso los intereses foráneos a la canalización del ahorro local-. Siguiendo a Ramos nuevamente (1980b; 14), "el problema fundamental es que las inversiones de capitales extranjeros en los países subdesarrollados generan un flujo inverso de transferencia de ganancias". Es decir, también por este lado existe una apropiación, por la clase dominante en las FSCD, de la plusvalía generada en estos países.

Igualmente afecta en este ámbito la dependencia tecnológica que aparece asociada a los dos planos de dependencia ya expuestos: la comercial, por la necesidad de importar una serie de insumos necesarios para el proceso productivo de contenido tecnológico inexistente en estos países - que además por razones de escala o de utilización de factores resulta inadecuada para el país receptor- y la financiera, a la que complementa como modalidad más sutil e impecable de apropiación de transferencia de ganancia. Obviamente, esta modalidad también tiene un importante efecto en la estructura productiva.

Sobre esta esfera también influye la dependencia cultural que se relaciona - en lo que aquí nos interesa- con lo que se ha dado en llamar "efecto demostración", consistente en la tendencia a la imitación de los patrones de consumo de las clases dominantes de los países desarrollados por parte de las clases dominantes de los países subdesarrollados, imitación que, a su vez, genera un efecto en cascada hacia los diferentes estratos de ingresos de estas sociedades. La implicación de este tipo de dependencia es importante en varios sentidos: por un lado, aumenta la tendencia al gasto suntuario disminuyendo la magnitud de ahorro disponible para inversión; por otro lado, supone una mayor propensión a importar productos de consumo, empleando en ello una porción importante de las escasas divisas disponibles (cuando no incentiva la instalación de empresas fabricantes de este tipo de productos, pro- 
vocando distorsiones aún mayores en la estructura productiva de estos países). Asimismo, esta dependencia cultural está muy estrechamente relacionada con los otros tipos de dependencia explicados previamente.

Todos estos espacios en que se manifiesta la dependencia afectan simultáneamente otros aspectos del proceso económico en su conjunto, por lo que serán retomados en su momento. Asimismo el significado global de la dependencia será brevemente discutido posteriormente.

En la esfera de la circulación, nos ocuparemos de los aspectos vinculados a la distribución, al intercambio y al consumo -o gasto- del producto.

En el ámbito de la distribución del producto —s decir, desde la óptica del ingreso-, lo primero que hay que señalar es la enorme concentración existente. Las transferencias netas de recursos al exterior, el desempleo y subempleo, el bajo nivel salarial -explicado, en parte, por la existencia de importantes contingentes de mano de obra cesante: el "ejército industrial de reserva"-, y la ineficaz $\longrightarrow$ inexistente- acción redistributiva del Estado -con ausencia de sistemas tributarios adecuados - se cuentan entre los factores explicativos de la escasa participación de los ingresos del trabajo - tanto directos como indirectos- en el ingreso y total. La principal consecuencia de esto es la no conformación de un mercado interno relevante a los efectos de posibilitar un tipo de crecimiento similar -de carácter autocentrado- al que ha tenido lugar en las FSCD10. Consecuencia de esto es la existencia de una estructura de consumo igualmente polarizada que impide la satisfacción de las necesidades básicas de una parte mayoritaria de la población. Estructura polarizada que se ve agravada por el "efecto demostración" referido anteriormente.

En cuanto al intercambio, éste se caracteriza por un deficiente funcionamiento de los mercados internos. De bienes (inflación de raíz estructural), de trabajo (desempleo y subempleo, bajos niveles salariales) y de dinero (tasas de interés altas que incentivan la orientación del ahorro a la especulación). Respecto a las relaciones con el exterior éstas también se ven presididas por el fenómeno de la dependencia en sus diferentes formas. Así, se genera una tenden- 
cia hacia el déficit crónico en la balanza comercial por la tendencia secular al deterioro de los términos de intercambio derivado de la inserción primario-exportadora de estos países y se produce, a través de las relaciones financieras, una transferencia neta de recursos desde ellos. Por lo tanto, nos encontramos con que el mal funcionamiento de los mercados impide una buena asignación de los recursos y con que la inserción exterior determina la pérdida de una parte importante del ya de por sí comparativamente reducido producto.

Finalmente, en lo que hace relación al gasto del producto - ya intercambiado y apropiado en las dos fases anteriores del proceso de circulación-, a la utilización del ingreso, a su uso desde la óptica del gasto, también aparecen algunos elementos peculiares de este tipo de formaciones sociales. El porcentaje destinado a consumo es más elevado que en las desarrolladas. Se explica por la excesiva propensión al consumo suntuario y el efecto negativo de la imitación de patrones de consumo exteriores - lo que constituye el "efecto demostración" ya citado, derivado de la dependencia en el terreno cultural-. Mas, sin embargo, eso va unido a la existencia de una mayoría de la población que no consigue ver garantizada la satisfacción de sus necesidades básicas. Por otro lado, la parte de la renta no consumida, el ahorro, es, lógicamente, considerablemente menor que en los países desarrollados, tanto en tamaño como en proporción. Además, en su canalización hacia inversión productiva se producen cuantiosos "escapes" como consecuencia de la dependencia exterior citada, de su orientación a uso especulativo, a fuga de capitales, etcétera. De modo que los recursos destinados a la acumulación son mucho más reducidos que en las FSCD, lo que limita sus posibilidades de crecimiento como se verá en el subsiguiente epígrafe.

En todo caso, lo más relevante de la esfera circulatoria, recién caracterizada en torno a una estructura de intercambio - tanto interior como exterior- distorsionada, a una estructura de distribución del ingreso de carácter concentradora y excluyente y a una estructura de gasto poco proclive a la acumulación, es que resulta funcional a la reproducción en el tiempo de estas formaciones sociales en su condición de subdesarrolladas. 
Conviene añadir una nota más respecto al fenómeno de la dependencia. Este supone - a través de sus diferentes mecanismos de actuación-, globalmente, una importante transferencia neta de recursos hacia la clase capitalista de las FSCD. Ello genera lo que algunos autores, como Marini (1973), han conceptualizado como "sobreexplotación", por cuanto la tasa de plusvalía o de explotación -i.e.: el trabajo no remunerado- es mayor que en las FSCD -diferencia que permite el "reparto" de su apropiación entre las clases dominantes de los países desarrollados y subdesarrollados-

Para concluir aquí con la cuestión de la dependencia, adelantaremos una discusión sobre la que se volverá más adelante. No es otra que el carácter de clase del fenómeno de la dependencia -y de ahí la virtud de la aportación que supone la conceptualización de Marini- De modo que, más allá de la medida en que dicho fenómeno colabore a explicar el subdesarrollo - en cuanto a su origen y, sobre todo, a la reproducción de esta situación crónica y estructural-, debe insertarse siempre en el marco de la lógica capitalista que lo determina, tanto de la formación social internamente como de su inserción exterior.

Resumiendo, podemos decir que el subdesarrollo afecta al conjunto de la estructura económica de estas formaciones sociales, de modo tal que la interrelación de sus distintas dimensiones, como veremos en el epígrafe siguiente, está en la explicación de su dinámica de reproducción en el tiempo.

\section{La reproducción del subdesarrollo: la lógica "perversa"}

I os distintos aspectos constitutivos del subdesarrollo explicados en el epígrafe anterior no son compartimentos estancos, sino que están mutuamente interrelacionados y retroalimentados. Por ello es en su análisis dinámico donde se nos ofrecen las mejores posibilidades de aprehender teóricamente el fenómeno.

El elemento central que explica la diferencia en la forma de reproducción económica del subdesarrollo respecto de los desarrollados se puede observar desde dos perspectivas.

Del lado de la oferta, el ciclo ahorro-inversión presenta una serie de deficiencias, descritas someramente en el apartado anterior, que impiden una tasa de acumulación elevada. Muy resu- 
midamente son las siguientes. En primer lugar, el ahorro es inferior al potencial: internamente, por una propensión a consumir alta $-\mathrm{y}$ porque, añadidamente, una parte importante de ese consumo lo representan bienes suntuarios que son producidos en el exterior con lo que ni siquiera son elementos de demanda para la producción nacional11 - y niveles de productividad bajos y, externamente, por saldo comercial inferior al potencial —consecuencia de tasas salariales más reducidas, etcétera-y por control del mercado mundial, crecientemente oligopolizado y oligopsonizado, extraño a estos países. $Y$, en segundo lugar, porque no todo el ahorro -como dijimos, escasose destina a inversión productiva porque una parte de él se orienta a la especulación y otra parte se "fuga" al exterior -dándose un flujo neto de recursos al exterior mediante repatriación de utilidades de extranjeros y evasión de los nacionales superior a los capitales recibidos-. A todo ello se le une el desaprovechamiento de recursos, los bajos niveles de productividad y otros elementos que, globalmente, explican que el crecimiento sea menor al potencial e incorpore sesgos importantes.

Del lado de la demanda, el carácter más marcadamente concentrador de las FSCS no permite la creación de un mercado interno fuerte, por lo que no juega un papel importante para la realización de la plusvalía, a diferencia de lo que sucede en las FSCD. Ello se convierte, nuevamente, en un obstáculo para la configuración de una estructura económica autocentrada con una base productiva más diversificada. Dicho de otro modo - y esto constituye un elemento central - en los países subdesarrollados la aguda concentración del ingreso es funcional a la reproducción de la situación de subdesarrollo. A diferencia de los desarrollados, en los que un cierto nivel de distribución es necesario para la realización del excedente.

De manera que, por el lado de la oferta, no se puede dar una acumulación de capital productivo tal que permita una progresiva ruptura con la vigente modalidad de integración de estos países en la economía mundial. Lo que, interrelacionado con la imposibilidad, por el lado de la demanda, de conformación de un mercado interno que sea estímulo suficiente para esa ruptura, prueba la inviabilidad de una hipótesis de desarrollo capitalista endógeno y autocentrado. Y de ahí, por tanto, la tendencia a la reproducción 
en el tiempo de la situación de subdesarrollo en estos países.

Otra forma de abordar la reproducción del subdesarrollo, útil para la comprensión de su lógica interna, es mediante el esquema sugerido por Baran (1959; 223 y ss.). En él, se plantea que el problema del subdesarrollo tiene que ver tanto con el tamaño del excedente como, sobre todo, con su uso -condicionado por la distribución del excedente-. Supone una importante aportación por la introducción que hace del rol que desempeña la distribución del ingreso - formulada como apropiación del excedentecomo variable explicativa del tipo de destino que se le da a éste.

Igualmente, nosotros podemos observar la reproducción de estas distorsiones considerando la estructura que describimos en el epígrafe anterior. Efectivamente, por un lado, tenemos que el producto es inferior al potencial -y con una participación sectorial distorsionada-. Después, que en la fase de intercambio el producto disponible se reduce como consecuencia del intercambio desigual entre países - además de que el mal funcionamiento de los mercados supone un fuerte desaprovechamiento de recursos e incide en las tendencias concentradoras del ingreso-. Por otro lado, la apropiación o distribución que se hace de este producto es tremendamente concentrada lo que impide la satisfacción de las necesidades del conjunto de la población -así como que ejerza un incentivo a la producción por el aumento de la demanda interna$y$, finalmente, en lo que constituye ya la última fase del proceso, tiende a favorecer la tendencia a un uso poco "acumulador" de recursos para la producción. Lo cual, nuevamente, condiciona el tamaño y estructura del producto y así sucesivamente. En toda esta cadena, la inserción de estos países en la economía mundial, concretada en su posición en la división internacional del trabajo, desempeña una importante función tanto por la detracción de recursos que supone como por las distorsiones que introduce en la esfera de la producción y de la circulación.

El resultado de todo esto es, de forma recurrente, un crecimiento inferior al potencial, inestable y distorsionado, una redistribución más regresiva del ingreso y una profundización de la inserción mundial dependiente. Rasgos que caracterizan a estas economías. $Y$ estos resultados tienden a reproducirse en el tiempo. A esta reproducción distorsionada que genera un crecimiento desequili- 
brado, sincopado e inestable - de modo que, conviene repetir una vez más, aunque en los desarrollados también se den estos rasgos, en los subdesarrollados se presentan de forma cuantitativa y cualitativamente más agudizada- es a lo que llamamos reproducción del subdesarrollo12.

En todo caso, un elemento que subyace, en tanto que desempeña un rol esencial en relación a lo planteado, es el mencionado carácter dependiente.

$\mathrm{Y}$, respecto a esto corresponde hacer una precisión que se nos antoja vital. Al hilo de ello basta recordar la fuerte polémica habida en lo tocante a si, efectivamente, existía dependencia y, en caso afirmativo, en qué medida sería entre naciones o entre clases y qué tipo de relación causal presentaría con el fenómeno del subdesarrollo. Hasta el momento, se ha manejado el concepto de dependencia sin delimitar su ubicación específica en el contexto teórico que se está definiendo. Sin ánimo de profundizar en la discusión, sí se explicitarán algunas cuestiones.

A pesar de considerar la variable clase como la unidad de análisis básica, ello no nos impide percibir la indudable existencia de formaciones sociales. Por cierto que no homogéneas, pero sí reales. Por tanto, plantear el análisis dialéctico situando la contradicción entre las clases antagónicas no impide, sino que requiere, contextualizarlo en el marco en que tiene lugar la concreción del modo de producción capitalista, es decir, en las formaciones sociales.

Pero es que, además, el fenómeno de la dependencia no es incompatible con el análisis en términos de clase, sino que es complementario, colaborando a la percepción del conflicto de clase -de la lucha de clases- a escala mundial. Efectivamente, no existe una explotación de unos países por otros que sea de carácter nacional. Dicha explotación es de carácter clasista. Por lo cual, no son los países desarrollados, sino su clase dominante, quién extrae una sobreplusvalía, y no es de los países subdesarrollados, sino de su clase dominada, de quién se extrae. Lo cual, está vinculado al concepto de sobreexplotación esbozado anteriormente. En todo caso, aunque se es plenamente consciente de la simplificación de lo expuesto especialmente en cuanto a que no hemos introducido en el esquema ni a la oligarquía de los países subdesarrollados ni a la clase trabajado- 
ra de los desarrollados, quienes obviamente también juegan un papel importante en esta cuestión- su pertinencia aquí obedece a que entendemos que puede ayudar a la visualización de la esencia del fenómeno de la dependencia en su interpretación tomando como unidad no tanto las formaciones sociales cuanto las clases, tal como desarrollan algunos autores dependentistas (como el propio Marini) ${ }^{13}$.

Ciertamente, el capitalismo en los países subdesarrollados es dependiente. Pero también es excluyente, concentrador, explotador, "hambreador", desarticulado, etcétera (rasgos algunos de los cuales son peculiares de las FSCS y otros no, y en diferentes medidas). En todo caso, lo verdaderamente relevante es su naturaleza capitalista. Y seguidamente las formas concretas de plasmarse en la dinámica social. Como impecablemente clarifica Cueva (1979; 78) cuando señala que "(...) conviene recordar, metodológicamente, que en la fórmula 'capitalismo dependiente' hay algo que es un sustantivo (capitalismo) y algo que es un adjetivo (dependiente) y que por lo tanto la esencia de nuestra problemática no puede descubrirse haciendo de la oposición capitalismo clásico/capitalismo dependiente el rasgo de mayor pertinencia, sino a partir de las leyes que rigen el funcionamiento de todo capitalismo".

4. ¿Un indicador de subdesarrollo?: los problemas de la medición

$\mathrm{H}$ asta el momento hemos analizado el fenómeno del subdesarrollo desde una perspectiva teórica, ocupándonos de su origen, su estructura y su reproducción. Sin embargo, por la importancia que tiene el problema de la "medición" lo abordaremos aunque más no sea sino muy brevemente.

Si nosotros hubiéramos partido de alguna de las visiones englobadas en lo que denominamos enfoque convencional, la tarea no sería difícil. Por ejemplo, si se identifica el desarrollo con crecimiento del producto, la medición puede hacerse con bastante precisión tomando el indicador que estima la tasa de variación del producto interior bruto -0 el nacional $u$ otro- en un período de tiempo determinado14. O si se le identifica con industrialización, midiendo la evolución de la participación del sector industrial en 
el total del producto, empleo, etcétera, o los índices de crecimiento de la producción del sector.

Sin embargo, como hemos expuesto en el artículo anterior de esta trilogía, entendemos que estas visiones mecánicas y parciales impiden un correcto acercamiento a la realidad social que tratan de comprender. De manera que nuestra tarea consiste en ver la forma de medir el subdesarrollo a partir de la conceptualización que de este fenómeno hemos llevado a cabo. Por ello, de lo que se trata es de optar por las variables que están en la esencia del subdesarrollo y no aquellas otras en que se manifiesta (como el bajo crecimiento del producto, inferior al potencial, o las elevadas tasas de inflación).

A partir de todo lo expuesto y del marco teórico planteado en los anteriores epígrafes, consideramos que no se puede hablar de un indicador que "mida" el subdesarrollo. Sin embargo, si hay tres elementos centrales que, en su interrelación, se revelan como constitutivos de la propia esencia del subdesarrollo. Y a los cuales se puede efectuar una aproximación empírica. Se trata de i) una aguda tendencia a la concentración y centralización del capital traducida en una creciente oligopolización de los mercados; ii) una fuerte dependencia externa (plasmada en términos de clase), y iii) una profunda concentración del ingreso que mantiene a una mayoría de la población ajena a los frutos del crecimiento económico (o, según el período, a una minoría ajena a las consecuencias de la crisis) lo que le lleva a una incapacidad crónica y estructural de satisfacer sus necesidades materiales básicas. $Y$ el corolario -verdadera esencia del subdesarrollo- de estos tres elementos, en su interrelación y mutua retroalimentación, es que este hecho es funcional a la reproducción del esquema en el tiempo.

\section{Recopilación}

1. El origen del subdesarrollo aparece asociado a la exparisión del modo de producción capitalista en la forma en que históricamente -de hecho- tuvo lugar, esto es, en una etapa primera de carácter comercial, y en una segunda de tipo financiero -conceptualizada como imperialista- Expansión que obedece a la lógica del capital en cuanto a la búsqueda de maximización de la ganancia. 
2. Una adecuada definición - como punto de partida- del subdesarrollo, ya citada, es la de Ramos (1980b; 16) para quien "como concepto teórico es la interrelación de una estructura interna distorsionada por la penetración del capitalismo exterior, con una serie de factores externos interconectados también entre sí y que son el resultado de la posición subordinada y asimétrica de los países subdesarrollados en la división internacional del trabajo, impuesta por los países desarrollados a lo largo de la historia". Fenómeno del subdesarrollo, conceptualizado como parte del proceso histórico global de desarrollo del capitalismo, que supone una estructura económica distorsionada y una transferencia de recursos de las FSCS a las FSCD - realizada mediante tasas mayores de explotación en aquellas-, cuya consecuencia fundamental es la de mantener a una parte mayoritaria de su población en unas muy deficientes condiciones de vida, que les impiden la satisfacción de sus necesidades básicas. De modo que la dimensión esencial del subdesarrollo en su expresión capitalista es su carácter clasista.

3. Los rasgos descriptivos de la estructura de las FSCS, dentro de su heterogeneidad, afectan a aspectos tanto de la esfera productiva como de la circulatoria, y encuentran un importante elemento explicativo en el papel desempeñado por los factores externos. Su condición sustancial es la de ser capitalistas si bien presentan peculiaridades importantes respecto a otras formaciones sociales también capitalistas, las desarrolladas.

4. La reproducción del subdesarrollo se puede observar tanto del lado de la oferta como del de la demanda -integrando aspectos internos y externos- por la funcionalidad a dicha reproducción de la interrelación entre ambas. El proceso económico presenta distorsiones en cada una de sus fases, lo que tiende a retroalimentar el alcance de esas distorsiones. El resultado de esto es un crecimiento inferior al potencial, inestable y distorsionado, una redistribución más regresiva del ingreso y una profundización de la inserción mundial dependiente que caracteriza a estas economías. Resultados estos que tienden a reproducirse en el tiempo, destacándose como la aguda concentración del ingreso es funcional a dicha reproducción.

5. En función de lo expuesto, el análisis empírico para los estudios de caso planteados en relación a la evolución de la condición 
de subdesarrollada de estas economías, se llevaría a cabo sobre la base de los tres elementos que están en la lógica constitutiva de la esencia del subdesarrollo. Ellos son i) la concentración y centralización del capital traducida en una creciente oligopolización de los mercados; ii) la fuerte dependencia externa (plasmada en términos de clase), y iii) la profunda concentración del ingreso que mantiene a una mayoría de la población ajena a los frutos del crecimiento económico (o, según el período, a una minoría ajena a las consecuencias de la crisis) lo que le lleva a una incapacidad crónica y estructural de satisfacer sus necesidades materiales básicas. Hecho éste que no sólo no es un obstáculo para la reproducción económica de estas sociedades en el tiempo sino que es funcional a ella.

\section{Referencias bibliográficas}

* Baran, Paul; La economía política del crecimiento, FCE, 1959.

* Frank, André Gunder; El desarrollo del subdesarrollo, Anagrama, 1971.

* Gill, Louis; Économie mondiale et impérialisme, Boréal Express, Québec, 1983.

* Marini, Ruy Mauro; Dialéctica de la dependencia, Serie Popular ERA, número 22, México, 1973.

- Marx; Karl; El Capital (Crítica de la Economía Política), Siglo XXI, 8 vols., Madrid, 1984; primera edición de la traducción: Buenos Aires, 1975.

* Palazuelos, Enrique (ed.); Las economías capitalistas durante el periodo de expansión 1945-1970, Akal, Madrid, 1986.

- Ramos, Antonio; El subdesarrollo: introducción metodologica, mimeo, UCM, 1979.

* _ _ Las fases de expansión del MPC, mimeo, UCM, 1980.

* - Las consecuencias de la expansión capitalista: la formación del subdesarrollo, mimeo, UCM, 1980.

* — ; Sobre la reproducción del subdesarrollo, mimeo, UCM, 1980.

- Sunkel, Osvaldo y PAZ, Pedro; El subdesarrollo latinoamericano y la teoría del desarrollo, Siglo XXI, México, 1970.

- Valenzuela F., José; ¿Qué es un patrón de acumulación?, Facultad de Economía, UNAM, México, 1990.

- _ C Critica del modelo neoliberal, Facultad de Economía, UNAM, México, 1991. 


\section{Notas}

1. El presente artículo se basa en el capítulo IV de ARRIZABALO, Xabier; Transnacionalización y subdesarrollo: Chile, 1973/1990 (Resultados económicos y significado histórico de la dictadura y el neoliberalismo), Tesis Docloral, Madrid, 1993.

2. Profesor de Economía de la Universidad Complutense de Madrid.

3. Por razones de concreción, en este artículo nos referiremos al subdesarrollo exclusivamente desde la disciplina de la economía. Razones de extensión nos impiden tratar otras dimensiones a las que, en todo caso, se hará referencia cuando ello sea pertinente. Por otra parte, citamos un texto bien sugerente respecto al tratamiento de esta temática: se trata de VALENZUELA (1990; especialmente el epígrafe titulado "Desarrollo y subdesarrollo: hipótesis de base", págs. 30 a 37).

4. Corresponde aclarar que la conceptualización del desarrollo capitalista como cíclico únicamente tiene sentido en cuanto a las tendencias de corto plazo (el "ciclo industrial", con duración de entre 7 y 10 años). No así en relación a las tendencias de largo plazo puesto que la existencia de fluctuaciones que dan lugar a períodos ascendentes y descendentes no asegura la perpetua reproducción del sistema en el tiempo. Esto es así debido a que la resolución de las crisis que reordenan el funcionamiento del sistema capitalista, no responde a ringún esquema lineal de tipo histórico, ni, por supuesto, a ningún mecanismo automático. Por ello, la sutileza respecto al uso del término cíclico no es tal sino que responde a una cuestión crucial. Para abundar en la cuestión, véase IZQUIERDO, Manuel P.; Los ciclos económicos largos. ¿Una explicación de la crisis?, Akal, Madrid, 1979.

5. Para expresarlo más gráficamente se puede pensar que la conocida expresión "Norte versus Sur" es errónea en tanto que obvia el hecho de que en el "Sur" también hay Norte y que en el "Norte" también hay Sur. Es un fenómeno similar al que pretende hacer creer que las fluctuaciones afectan igual a todos los sectores sociales, cuando se constata como, tanto en el "Norte" como en el "Sur", de la misma manera que hay mayorías que permanecen ajenas a los frutos del crecimiento económico cuando este realmente existe, también hay minorías que permanecen ajenas a las consecuencias de la ausencia de este crecimiento, de la crisis, es decir, que hay sectores que no pierden en las crisis y otros sectores -los otros- que no ganan en los auges. Todo esto es inherente a la lógica de funcionamiento capitalista que conforma clases cuyos intereses son antagónicos.

6. Manejar el concepto de dependencia en cuanto instrumento de análisis no implica, por nuestra parte, ningún posicionamiento teórico dependentista, en cuanto variable explicativa del subdesarrollo puesto que entendemos que la explicación se encuentra en la interrelación de los elementos citados anteriormente. $Y$ menos aún esbozo alguno de análisis en términos nacionales, por cuanto que situamos el principal conflicto no en la competencia entre naciones sino, a escala mundial - y nacional-, entre clases, es decir, en la lucha de clases. En todo caso, más adelante abordaremos con mayor detenimiento este tema. 
7. De nuevo conviene aclarar que la división en distintos planos se hace con fines de hacer más clara la exposición, ya que en la realidad todos se encuentran absolutamente interrelacionados.

8. No obstante, es oportuno señalar que, en este punto, como en muchos otros, las diferencias entre países -y entre los distintos períodos- son abismales.

9. Sobre ello se volverá más adelante. En todo caso, para entender este punto es pertinente retomar la idea de la "alianza" entre la clase capitalista de las FSCD y la oligarquía de las FSCS. Siempre en el marco conceptual de la lucha de clases.

10. Lo cual, nuevamente interesa puntualizarlo, no implica ningún "piropo" a la evolución de estos países en donde, también, aparecen bastantes de los factores reseñados respecto a la concentración del ingreso, si bien en una medida radicalmente distinta.

11. Véase en el apartado anterior las implicaciones de la dependencia de patrones de consumo.

12. Lo que FRANK (1971) denominó "desarrollo del subdesarrollo".

13. Ciertamente, el esquema sugerido de apropiación de una parte de la plusvalía generada en las FSCS por parte de la clase dominada de las desarrolladas es simplista en exceso. No obstante, su inclusión aquí obedece a la claridad que puede aportar. Además, hay otra razón que apoya dicha inclusión. Se trata de la observación de cómo, tendencialmente y en términos de magnitud, la pérdida de participación de los ingresos del trabajo en el producto total de las FSCS coincide significativamente en el tiempo con el incremento de la transferencia neta de recursos de estas sociedades a las desarrolladas.

14. En realidad, hasta la precisión de estos indicadores es discutible. El cuestionamiento se hace sobre la base de hechos vinculados a distorsiones en: i) los contenidos del indicador tales como que "en las estadísticas de renta nacional el nacimiento de un ternero representa un incremento del nivel de vida, mientras que el nacimiento de un niño supone un descenso" (BAUER, P.T.; Crítica de la teoría del desartollo, Orbis, Barcelona, 1983, pág. 70) o como que la depreciación del capital natural por una sobreexplotación de los recursos naturales no sea tenida en cuenta; ii) los objetivos de desarrollo definidos con base en los indicadores que lo "miden", de modo que "como es bien sabido, las dificultades estadísticas hacen imposible construir un índice del producto nacional bruto que registre de forma fiable los cambios de nuestro bienestar material (...) Pero una vez construido un índice, con todas sus imperfecciones, asume importancia propia. Para contrarrestar campañas políticas (...) los gobiernos adoptan acciones diseñadas para la elevación de las cifras del indice en vez de para la elevación del bienestar de la población" (LINDER, Staffan B.; The Harried Leisure Class, New York, 1970, pág. 139; tomado de BAUER, P.T. -1983; pág. 73-), y, iii) la elaboración de las estadísticas. Por otra parte, incluso algunos organismos multilaterales se han ido preocupando del tema con la inclusión de otros aspectos en los indicadores para la medición del subdesarrollo. Es el caso del Banco Mundial al tratar el tema de la pobreza o del Programa de Naciones Unidas para el Desarrollo con su Indice del Desarrollo Humano. Si bien no aportan gran cosa a la discusión, el mero hecho de que lo consideren ya es de por sí significativo. 